\title{
Avaliação das Habilidades Sociais em Adultos Idosos e Adultos
}

\author{
Jucelaine Bier Di Domenico-Grazziotin ${ }^{1}$ \\ Silvana Alba Scortegagna \\ Programa de Pós-Graduação em Envelhecimento Humano da Universidade de Passo Fundo, \\ Passo Fundo, RS, Brasil
}

\begin{abstract}
Resumo
A competência social consiste em uma das condições indispensáveis para a atribuição de saúde mental. Este estudo objetivou avaliar o repertório de Habilidades Sociais (HS) em adultos idosos e adultos e verificar se adultos idosos, especialmente as faixas etárias mais velhas, apresentavam um repertório menos desenvolvido em HS. Participaram desse estudo 135 indivíduos saudáveis com idades entre 18 e 83 anos, distribuídos em dois grupos: um grupo de adultos idosos com 61 participantes, e outro grupo de adultos, com 74 indivíduos. Os instrumentos utilizados foram um protocolo de caracterização sociodemográfica e de saúde, o Inventário de Habilidades Sociais-Del Prette (IHS) para adultos e o IHS adaptado para idosos. Houve correlações significativas e negativas entre a idade avançada dos participantes e as HS no escore geral GIHS (Escore Geral do Inventario de Habilidades Sociais; $r=0,20$ ), no afeto positivo F2 $(r=0,249)$, na conversação e desenvoltura social F3 $(r=0,259) \mathrm{e}$, no autocontrole da agressividade F5 $(r=0,490)$. Os adultos idosos, apresentaram interações sociais mais restritas e um repertório nas HS menos desenvolvido, em comparação aos adultos. O desenvolvimento de políticas públicas que valorizem as HS para auxiliar a sustentar o bem-estar na idade adulta mais velha é uma necessidade do contexto socioeconômico e cultural brasileiro.
\end{abstract}

Palavras-chave: Avaliação psicológica, envelhecimento humano, relacionamento interpessoal, cultura.

\section{Evaluation of Social Skills in Older Adults and Adults}

\begin{abstract}
Social competence comprises one of the indispensable conditions for mental health. The aim of this study was to evaluate the Social Skills (SS) repertoire in older adults and adults to verify whether older adults, especially those of the older age groups, present a less developed SS repertoire. Participants of this study were 135 healthy subjects aged 18 to 83 years, divided into two groups: one group (Older Adults Group [OAG]) consisting of 61 older adults, and the other group (Adults Group [AG]) consisting of 74 adults. The instruments used were a sociodemographic and health characterization questionnaire, the Social Skills Inventory-Del Prette (SSI) for adults and the SSI adapted for older adults. There were significant and negative correlations between the age of the participants and the overall SS score GSSI (Overall Score Social Skills Inventory; $r=.20)$, in the positive affect F2 $(r=.249)$, in the conversation and social confidence F3 ( $r=.259)$ and in self-control of aggressiveness F5 $(r=.490)$ factors. The OAG
\end{abstract}

Endereço para correspondência: Universidade de Passo Fundo, Programa de Pós-Graduação em Envelhecimento Humano, BR 285 - Cx. Postal 611, Bairro São José, Passo Fundo, RS, Brasil 99052-900. E-mail: jucelainegraz@ terra.com.bre silvanalba@upf.br 
presented more restricted social interactions and a less developed SS repertoire, compared to the AG. The development of public policies that valorize SS to help sustain the well-being of older adults is a necessity in the Brazilian socioeconomic and cultural context.

Keywords: Assessment psychological, elderly, interpersonal relationship, cultura.

\section{Evaluación de las Habilidades Sociales en los Adultos Mayores y los Adultos}

\section{Resumen}

La competencia social consiste en dar condiciones indispensables para la atribución de la salud mental. El estudio objetivó evaluar el repertorio de Habilidades Sociales (HS) en adultos mayores y adultos y verificar si los ancianos, de manera especial los grupos de edades más avanzadas, presentaban un repertorio menos desarrollado en HS. Participaron 135 personas sanas, con edades entre 18 y 83 años, seleccionados en dos grupos: un grupo de 61 adultos mayores, y otro grupo de 74 personas adultos. Los instrumentos utilizados fueron preguntas de caracterización socio demográfica y de salud, el Inventario de Habilidades Sociales-Del Prette (IHS) para adultos y, para los mayores, ha sido adaptado el IHS. Hubo correlación significativa negativa entre la edad avanzada de los participantes y las HS en la puntuación general GIHS (Escore Geral do Inventario de Habilidades Sociais; $r=.20$ ), en el afecto positivo F2 $(r=.249)$, en la conversación y desenvoltura sociales F3 $(r=.259)$, y en el autocontrol de la agresividad F5 $(r=.490)$. Los adultos mayores presentaron interacciones sociales más restrictas y un repertorio en las HS menos desarrollado, en comparación a los adultos. El desarrollo de políticas públicas que valoren las HS para auxiliar y para mantener el bienestar en la edad adulta mayor es una necesidad del contexto socioeconómico y cultural brasileño.

Palabras-clave: Evaluación psicológica, envejecimiento humano, relación interpersonal, cultura.

Em todo mundo há uma transição do processo demográfico que acena para um predomínio de populações mais velhas. A proporção de pessoas com 60 anos e mais deve duplicar e atingir dois bilhões em 2050 (United Nations, 2012). No Brasil, estimativas projetam que o número de pessoas idosos até 2025 será superior a 30 milhões (Instituto Brasileiro de Geografia e Estatística [IBGE], 2010).

O aumento acentuado de idosos traz consequências para a sociedade no que diz respeito a saúde, educação, trabalho, prestação de serviços, e desenvolvimento de pesquisas (American Psychological Association, 2014). Deste modo, é necessário buscar os fatores determinantes das condições de saúde e doença que incidem no processo de envelhecimento e na velhice, principalmente nos países emergentes como o Brasil em que os estudos são incipientes (Bastos-Formighieri \& Pasian, 2012). Entre estes fatores encontra-se a Habilidade Social (HS) que ilustra a qualidade dos relacionamentos interpessoais, no que se refere à comunicação, civilidade, assertividade, empatia, trabalho, bem como a expressão de sentimentos positivos (Del Prette \& Del Prette, 2011, 2013).

As HS são classes de comportamento que existem em um repertório do indivíduo e contribuem para uma conduta socialmente competente. Tal comportamento implica em realizar funções esperadas pela sociedade, que pode ser: liderança, coordenação de grupos, administrar o stress, conflitos interpessoais, intrapessoais e intergrupais (Del Prette \& Del Prette, 2001, 2013).

Um repertório bem desenvolvido em HS relaciona-se a maior satisfação pessoal, profissional e, consequentemente, contribui para promover a qualidade de vida e a saúde mental (Caballo, 2008; Del Prette \& Del Prette, 2005, 2013; Del Prette, Falcone, \& Murta, 2013). Os transtornos e problemas psicológicos associados aos déficits nas HS incluem o stress, a solidão, a 
depressão, um maior risco de suicídio, a ansiedade, os conflitos conjugais (Angélico, Crippa, \& Loureiro, 2012; Carneiro, Falcone, Clark, Del Prette, \& Del Prette, 2007; Del Prette et al., 2013; Pinto \& Barham, 2014; Schlösser, Rosa, \& More, 2014). A presença desses problemas pode ser influenciada pelos tipos de interações sociais que as pessoas estabelecem no decorrer da vida, e podem ser decorrentes de fatores pessoais e contextuais (Del Prette \& Del Prette, 2005, 2011).

Relativo às questões contextuais, as HS podem ser aprendidas e culturalmente determinadas, evidenciando assim a importância das oportunidades advindas do contexto ambiental e social (Del Prette \& Del Prette, 2005). O ambiente reforça ou não, os comportamentos sociais, e possibilita a aprendizagem de novas habilidades por meio de observação e de interações. Os elementos ambientais incluem a idade e a etapa do desenvolvimento, o gênero, a escolaridade, a situação familiar, cultural, socioeconômica e ocupacional.

As interações e as atividades sociais podem impulsionar a expansão de redes de apoio social e ajudar no enfrentamento de várias demandas interpessoais, e contribuir para a eficácia pessoal, para a constituição de uma identidade positiva, e uma boa autoestima. O desenvolvimento da assertividade, por exemplo, que supõe a expressão de direitos, pensamentos, sentimentos e crenças de forma a não violar o direito dos outros, pode ser extremamente relevante (Braz, Del Prette, \& Del Prette, 2011).

Embora as respostas ao envelhecimento ocorram de maneira diferenciada para cada indivíduo, a pouca valorização do adulto idoso, dos aspectos positivos como a maturidade e a sabedoria, na cultura ocidental (Baltes \& Baltes, 1990; Garcia-Santos, Almeida, \& Werlang, 2012), aliada ao incremento dos aspectos negativos como perdas decorrentes da senescência, pode levá-lo a apresentar um menor investimento nas relações interpessoais, socioafetivas e produtivas (McAdams \& Olson, 2010; Neri \& Vieira, 2013; O’Brien, Konrath, Gruhn, \& Hagen, 2013). Especialmente os adultos de 80 anos e mais idade, tendem a apresentar mais limita- ções físicas e restrições no engajamento social (Neri \& Vieira, 2013). A autora destaca que o envolvimento em atividades sociais traz benefícios para a cognição, a saúde, a longevidade e a funcionalidade, favorece o sentir-se útil, o senso de pertencimento e os intercâmbios de ajuda, o que deve ser incentivado.

É nesse contexto que se insere o campo das HS, como classes de comportamentos que contribuem na promoção da saúde, na superação das dificuldades, na intervenção clínica, educativa e na prevenção de transtornos de personalidade (Angélico et al., 2012; Braz, Cômodo, Del Prette, Del Prette, \& Fontaine, 2013; Braz et al., 2011; Carneiro et al., 2007; Del Prette et al., 2013; Di Domenico-Grazziotin \& Scortegagna, 2012, 2013; Feitosa, Del Prette, \& Del Prette, 2012; Guilland \& Monteiro, 2010; Neri \& Vieira, 2013; Pinto \& Barham, 2014). A correlação entre HS e bem-estar psicológico, sugerindo que quanto maior o índice de HS, melhor a condição de saúde e menor a probabilidade de satisfazer os critérios de rastreamento de indicadores para a doença, foi apontada em pesquisas com adultos não idosos (Angélico et al., 2012; Guilland \& Monteiro, 2010; Pinto \& Barham, 2014).

Outros estudos com adolescentes e adultos também evidenciaram a associação entre HS, relacionamento interpessoal no ambiente escolar e de trabalho, reafirmando que indivíduos com escores mais elevados nas HS interagem mais, e apresentam maior rendimento acadêmico (Di Domenico-Grazziotin \& Scortegagna, 2012, 2013; Feitosa et al., 2012). Já, com a população adulta idosa, os estudos são escassos e as HS tem sido relacionadas à qualidade de vida, ao apoio social e à depressão, indicando que as deficiências em tais habilidades podem constituir um fator de vulnerabilidade para a baixa qualidade de vida (Carneiro \& Falcone, 2013; Carneiro et al., 2007).

Diante destes achados, alguns pesquisadores demonstraram a possibilidade de se intervir para desenvolver o repertório em HS. Braz et al. (2011) avaliaram os efeitos de um programa de treinamento das HS assertivas com 15 adultos idosos, com 60 anos e mais, distribuídos, em dois grupos. O primeiro, um grupo experimental 
com sete mulheres e um homem, média de idade 67,5 anos, e o segundo, um grupo controle, com seis mulheres e um homem, média de idade 65,5. Os autores utilizaram o Inventário de Habilidades Sociais para idosos (IHSI-Del Prette), com a adaptação de algumas frases do Inventário de Habilidades Sociais-Del Prette (IHS-Del Prette) para indivíduos com 60 anos de idade e mais (Carneiro et al., 2007). Os resultados indicaram diferenças significativas do pré-teste e pós-teste, com um aumento no escore geral das HS neste último.

Posteriormente, Braz et al. (2013) realizaram dois estudos com o objetivo de investigar a correlação entre o repertório de HS entre pais idosos e filhos adultos, e a influência da qualidade da relação entre eles no repertório destas habilidades. O primeiro estudo avaliou 142 díades, pais adultos e filhos adolescentes. O segundo estudo, investigou a relação entre 77 pais idosos e 107 filhos adultos. Os instrumentos utilizados foram: o Inventário de Habilidades Sociais para Adolescentes (IHSA-Del Prette), o IHS-Del Prette e, o IHSI adaptado para adultos idosos (Carneiro et al., 2007). Os resultados mostraram semelhanças para 29 das 38 HS avaliadas entre eles e, também, evidências da transmissão intergeracional de várias classes de HS, especialmente entre mães e filhos adolescentes.

$\mathrm{Na}$ busca em mapear outras variáveis que possam intervir no desenvolvimento das HS, Neri e Vieira (2013) analisaram as relações entre envolvimento e suporte social em adultos idosos, considerando a idade, o gênero, a escolaridade e a localidade de residência. Participaram do estudo 1.451 adultos idosos sem déficit cognitivo sugestivo de demência. Os instrumentos utilizados foram o Mini Exame do Estado Mental (MEEM), um questionário de desempenho de atividades avançadas de vida diária e o suporte social percebido. Entre os resultados, o envolvimento social foi maior entre as mulheres, nos adultos idosos mais jovens entre 65 a 74 anos de idade, com nível mais alto de escolaridade e residentes em localidades economicamente mais desenvolvidas. Foram observadas correlações negativas entre envolvimento social e idade.
Considerando que as HS podem estar associada à saúde mental, a um bom relacionamento interpessoal e à qualidade de vida (Angélico et al., 2012; Braz et al., 2011; Carneiro \& Falcone, 2013; Carneiro et al., 2007; Guilland \& Monteiro, 2010; Neri \& Vieira, 2013; Pinto \& Barham, 2014), o objetivo do presente estudo foi avaliar o repertório de HS em adultos idosos e adultos e verificar se adultos idosos, especialmente as faixas etárias mais velhas, apresentavam um repertório menos desenvolvido em HS quando comparados com adultos não idosos.

Se indivíduos idosos podem apresentar declínio e limitações nos investimentos das relações interpessoais e sociais (Braz et al., 2011; McAdams \& Olson, 2010; Neri \& Vieira, 2013; O'Brien et al., 2013), tem-se como hipótese que o avanço da idade, aliada às condições sociodemográficas e culturais, poderão propiciar um rebaixamento significativo nos escores dos fatores F1 (Enfrentamento e autoafirmação com risco), F2 (Autoafirmação na expressão de afeto positivo), F3 (Conversação e desenvoltura social), F4 (Autoexposição a desconhecidos ou a situações novas) e F5 (Autocontrole da agressividade em situações aversivas) e no escore geral - GIHS (Escore Geral do Inventario de Habilidades Sociais; Del Prette \& Del Prette, 2005).

\section{Método}

\section{Participantes}

Participaram desse estudo 135 pessoas com idade entre 18 a 83 anos, selecionados de forma não aleatória e distribuídas em dois grupos. $\mathrm{O}$ primeiro grupo foi composto por 61 adultos idosos com 60 anos e mais ( $45 \%$ da amostra), a média de idade foi de 68,75 anos, $(D P=6,36)$, sendo 43 mulheres e 18 homens, a média de escolaridade foi de 7 anos, $(D P=2,33)$ e de remuneração foi de 1,7 salários mínimos, $(D P=0,67)$, casados (71\%), procedentes de centros de convivência. $O$ segundo grupo foi composto por 74 indivíduos, menores de 60 anos (55\% da amostra), a média de idade foi de 37 anos $(D P=14,3)$, sendo 46 mulheres e 28 homens, a média de escolaridade foi de 10 anos $(D P=2,03)$ e, de remuneração foi de 1,8 salários mínimos $(D P=0,53)$, casados 
(53\%), provenientes de estabelecimentos comerciais, que trabalhavam com atividades de atendimento ao público.

Como critérios de inclusão e de exclusão, para os adultos idosos, foram considerados aptos para o estudo, aqueles com idades de 60 anos e mais, que participavam de atividades em grupos de convivência. Para os adultos, considerou-se aqueles entre 18 a 59 anos de idade que trabalhavam com atividades de atendimento direto ao público em estabelecimentos comerciais. Para ambos os grupos, a participação na pesquisa foi condicionada a não apresentarem: (a) histórico de tratamento psiquiátrico e/ou de doenças que comprometessem a capacidade para a realização de atividades cognitivas ou físicas; (b) histórico de alguma perda importante que acarretou sofrimento, nos últimos seis meses. Esses dados foram obtidos por meio da administração do protocolo de caracterização sociodemográfica e de saúde.

\section{Instrumentos}

Protocolo de Caracterização Sociodemográfica e de Saúde. Com o objetivo de verificar os critérios de inclusão ou exclusão, e caracterizar a amostra. $\mathrm{O}$ instrumento constitui-se de 12 questões fechadas sobre idade, gênero, estado civil, profissão, escolaridade, condições socioeconômicas e de saúde (presença de doenças, tratamento médico/ psiquiátrico, perdas nos últimos seis meses).

Inventário de Habilidades Sociais (IHS; Del Prette \& Del Prette, 2001). Este instrumento visa avaliar o repertório de habilidades sociais usualmente requerido em situações cotidianas (trabalho, família, amigos) por meio de 38 itens, que descrevem uma relação interpessoal e um possível desfecho. Na resposta a cada item, o respondente deve estimar a frequência com que reage a uma situação específica, em uma escala tipo Likert, que varia de 0 (nunca ou raramente) a 4 (sempre ou quase sempre), de 5 pontos, com um escore total entre 0 e 152 . Apresenta a seguinte estrutura fatorial: (a) Fator 1 (F1): Enfrentamento e autoafirmação com risco (composto pelos itens $1,5,7,11,12,14,15,16,20$, 21, 29); (b) Fator 2 (F2): Autoafirmação na ex- pressão de afeto positivo (itens $3,6,8,10,28$, 30, 35); (c) Fator 3 (F3): Conversação e desenvoltura social (itens 13, 17, 19, 22, 24, 36, 37); (d) Fator 4 (F4): Autoexposição a desconhecidos ou a situações novas (itens 9, 14, 23, 26); (e) Fator 5 (F5): Autocontrole da agressividade em situações aversivas (itens 18, 31, 38).

$\mathrm{Na}$ interpretação dos resultados, quanto menor o percentil, mais o indivíduo tende a apresentar um déficit em seu repertório de HS e, quanto mais alto o percentil, melhor o seu repertório em HS. Os valores de 0 a 20 são considerados inferiores (I) e, nesse caso, apontam déficits em HS. Os percentis de 25 a 45 são considerados médios inferiores (MI), e os próximos a 50 , médios $(\mathrm{M})$, analisados como bom repertório. Valores de 55 a 75 são avaliados como médios superiores (MS), interpretados como repertório elaborado, e, de 80 em diante, superiores (S), correspondendo a um repertório bastante elaborado.

$\mathrm{Na}$ análise das propriedades psicométricas, o IHS-Del Prete apresentou um padrão de confiabilidade e de consistência interna satisfatória com um alfa de Cronbach de 0,75; estabilidade teste-reteste $(r=0,90 ; p=0,001)$ e validade concomitante com o Inventário de Rathus ( $r=0,79 ; p=0,01$; Bandeira, Costa, Del Prette, Del Prette, \& Gerk-Carneiro, 2000). Como este instrumento foi validado para a população universitária de 18 a 25 anos, realizou-se para este estudo a adaptação das questões de número: $2 ; 5 ; 8 ; 10 ; 11 ; 13 ; 14 ; 15 ; 17 ; 18$; $22 ; 29 ; 34$ e 38 para a faixa de idade de 60 anos e mais (Carneiro et al., 2007).

Desta forma, o inventário de HS para Idosos, consiste em uma versão do Inventário de HS (IHS-Del Prette) e as propriedades psicométricas são as mesmas estabelecidas na versão original (Del Prette \& Del Prette, 2001). As adaptações não alteram a estrutura das questões mas alguns termos são substituídos para adaptarem-se a realidade da população idosa, exemplo: questão 2- Os termos "pais" e "irmãos mais velhos" foram substituídos por "filhos", "irmão" e "sobrinhos"; na questão 5- Foi acrescentado o termo "parente"; na questão 8- "Mesmo junto a conhecidos da escola ou do trabalho" foi 
modificado para "mesmo junto a conhecidos" (Carneiro et al., 2007).

\section{Procedimentos}

De posse da carta de autorização das instituições, e da aprovação do estudo pelo Comitê de Ética em Pesquisa da Universidade de Passo Fundo (UPF), protocolo de número 076/2009, iniciou-se a coleta dos dados. Os adultos idosos foram recrutados em um centro de convivência com aproximadamente 250 integrantes. Após serem expostos os objetivos e procedimentos da pesquisa aos participantes, durante a realização dos grupos de convivência, os que manifestaram interesse em fazer parte do estudo foram encaminhados pelos coordenadores dos grupos.

Os adultos foram recrutados em dois estabelecimentos comerciais que possuem cerca de 130 colaboradores, situados no centro da cidade. Após a exposição dos objetivos e procedimentos do estudo aos funcionários que exerciam funções de atendimento direto ao público, os que manifestaram interesse em compor o estudo foram encaminhados pelo gerente do setor comercial.

Os participantes do presente estudo assinaram o Termo de Consentimento Livre e Esclarecido (TCLE) e responderam aos instrumentos após o rapport, individualmente, em uma sessão de aproximadamente 35 minutos, nas dependências das instituições. A aplicação dos instrumen- tos foi realizado pela autora principal do estudo e, por uma aluna do Curso de Especialização em Psicologia da Saúde da UPF, devidamente treinada. Primeiramente foi administrado o protocolo de caracterização sociodemográfica e de saúde (critérios de exclusão/exclusão) e, na sequencia, o IHS-Del Prette para adultos com menos de 60 anos e o IHS adaptado para os adultos idosos com 60 anos e mais. Para os idosos a administração foi realizada de forma assistida, por solicitação destes, e para os participantes com menos de 60 anos o instrumento foi autoaplicado.

Os dados foram gerados de acordo com o manual do IHS-Del Prette adaptado à população de idosos. Para análise dos resultados, foi utilizado o programa estatístico SSPS, versão 18.0. Após a realização da estatística descritiva realizou-se a associação entre os escores do instrumento e a idade dos participantes por meio da correlação linear de Pearson $(p \leq 0,05)$. O teste paramétrico escolhido foi devido as variáveis terem seguido uma distribuição normal, resultado obtido após a aplicação do teste Kolmogorov Smirnov.

\section{Resultados}

Na descrição dos resultados, serão apresentadas, primeiramente, as estatísticas descritivas do IHS- Del Prette. Na sequência, será demonstrada a correlação de Pearson com o IHS-Del Prette e a idade dos participantes.

Tabela 1

Média e Desvios Padrão do IHS-Del Prette dos Participantes

\begin{tabular}{ccccc}
\hline & $\begin{array}{c}\text { Adultos Idosos } \\
60 \text { anos e mais } \\
N=61\end{array}$ & \multicolumn{2}{c}{$\begin{array}{c}\text { Adultos } \\
\text { Menos de } 60 \text { anos } \\
N=74\end{array}$} \\
\hline IHS & Média & $D P$ & Média & $D P$ \\
GIHS & 41,38 & 31,11 & 51,82 & 30,11 \\
F1IHS & 54,52 & 32,68 & 46,50 & 31,49 \\
F2IHS & 47,07 & 32,49 & 60,84 & 30,16 \\
F3IHS & 41,20 & 36,10 & 55,69 & 33,99 \\
F4IHS & 36,04 & 23,11 & 43,93 & 25,26 \\
F5IHS & 34,85 & 19,50 & 57,04 & 24,23 \\
\hline
\end{tabular}

Nota . GIHS = Escore Geral do Inventario de Habilidades Sociais. Na média, consta valores obtidos em percentil do IHS-Del Prette informatizado. 
Os dados exibidos na Tabela 1 permitem observar que os participantes idosos evidenciaram resultados um pouco abaixo da média para o escore geral de habilidades sociais, para os fatores comunicação e desenvoltura social (F3), autoexposição a desconhecidos ou a situações novas (F4) e autocontrole da agressividade em situações aversivas (F5). Porém, no enfrentamento e autoafirmação com risco (F1) e na autoafirmação na expressão de afeto positivo (F2), os resultados mantiveram-se na média.

Os adultos apresentaram resultados na média para o escore geral de habilidades sociais (GIHS) e para os fatores: o enfrentamento e a autoafirmação com risco (F1) e a comunicação e desenvoltura social (F3). Obtiveram destaque, nos fatores de autoafirmação na expressão de afeto positivo (F2) e de autocontrole da agressividade em situações aversivas (F5), com escores acima da média. Contudo, constata-se que, na autoexposição a desconhecidos ou a situações novas (F4), os escores ficaram abaixo da média, o que sugere dificuldades nessa área.

Esses resultados sugerem que as $\mathrm{HS}$ em termos gerais, e nos respectivos fatores, encontram-se menos desenvolvidas nos participantes idosos, quando comparadas às habilidades dos adultos não idosos. Na sequência, será demonstrado os resultados das análises de correlação de Pearson entre o IHS-Del Prette e a idade dos participantes.

Tabela 2

Correlações do IHS-Del Prette com as Idades dos Participantes

\begin{tabular}{ccccccc}
\hline Idade & GIHS & F1IHS & F2IHS & F3IHS & F4IHS & F5IHS \\
\hline Correlação de Pearson & $-0,201^{*}$ & 0,134 & $-0,249^{* *}$ & $-0,259^{* *}$ & $-0,186^{*}$ & $-0,490^{* *}$ \\
Sig. (2-tailed) & 0,020 & 0,122 & 0,004 & 0,002 & 0,031 & 0,000 \\
\hline
\end{tabular}

* Significativo $p \leq 0,05 ; * *$ significativo $p \leq 0,01$.

Observa-se, na Tabela 2, correlações estatisticamente significativas de forma negativa e baixa entre a maior idade e repertório de habilidades sociais gerais (GIHS), autoafirmação na expressão de afeto positivo (F2) e, comunicação e desenvoltura social (F3). Nota-se uma correlação significativa negativa e moderada entre a maior idade e o autocontrole da agressividade em situações aversivas (F5). Esses dados corroboram os achados da Tabela 1 e serão discutidos a seguir.

\section{Discussão}

O presente estudo buscou avaliar o repertório de HS em adultos idosos e adultos e verificar se adultos idosos, especialmente as faixas etárias mais velhas, apresentavam um repertório menos desenvolvido em HS quando comparados com adultos. Entre os resultados do escore geral GIHS verificou-se que os adultos idosos apresentaram repertório médio inferior, e os adultos permaneceram na média e houve uma correla- ção significativamente baixa e negativa do GIHS com a idade $(r=0,201)$. Estes dados demonstram a possibilidade das HS em geral, que retratam a capacidade de empatia, de expressividade emocional e podem promover os relacionamentos interpessoais e o bem-estar nos diversos contextos (Angélico et al., 2012; Del Prette \& Del Prette, 2005, 2011; Di Domenico-Grazziotin \& Scortegagna, 2012, 2013; Feitosa et al., 2012; Guilland \& Monteiro, 2010) apresentarem-se menos desenvolvida à medida que a idade avança.

Esses achados vêm ao encontro da hipótese inicial deste estudo, e demonstram concordância com estudos prévios que afirmam que, embora o envelhecimento ocorra de forma heterogênea, pode haver nos idosos um menor investimento nas relações interpessoais, sociais e produtivas (McAdams \& Olson, 2010; O'Brien et al., 2013). Isto pode ser decorrente, por exemplo, dos conflitos da fase da velhice (Braz et al., 2011; Del Prette \& Del Prette, 2011). É comum nas idades mais avançadas que os idosos vivenciem situações que requeiram o enfrenta- 
mento das perdas de pessoas próximas, o ajuste das condições socioeconômicas e do status social mais desfavorável, especialmente nas culturas ocidentais (Neri \& Vieira, 2013).

No F2 (autoafirmação na expressão de sentimentos positivos) que envolve afirmação da autoestima, fazer amizades, elogiar e agradecer amabilidades (Del Prette \& Del Prette, 2005), verifica-se que as pessoas idosas deste estudo, demonstraram um escore médio. A correlação significativa de forma negativa e baixa $(r=0,249)$ baliza uma tendência de que a autoafirmação na expressão de afeto positivo (F2) apresentese menos desenvolvida à medida que a idade avança. Esse achado, igualmente, vem ao encontro da hipótese inicial deste estudo, de tal capacidade diminuir em idosos com idades mais avançadas, e corrobora outros estudos (McAdams \& Olson, 2010; Neri \& Vieira, 2013; O'Brien et al., 2013).

É importante ponderar que a diminuição no campo afetivo não resulta somente do incremento da idade isoladamente, mas de um contexto multifatorial (condições de saúde, econômicas, familiares, educacionais e culturais) que envolve o envelhecimento (Neri \& Vieira, 2013; Schlösser et al., 2014). Para se conhecer melhor a extensão destes fatores e as interferências no final da vida, é necessário o incremento de pesquisas com adultos idosos, um segmento da população ainda pouco estudado (American Psychological Association, 2014; United Nations, 2012).

No Brasil, as condições oferecidas a esta população, os cuidados em relação à saúde, as oportunidades de trabalho e de inserção social são, ainda, muito incipientes (IBGE, 2010; Neri \& Vieira, 2013). Estas preocupações se refletem nas pesquisas que buscam conhecer as causas e os determinantes de uma velhice bem-sucedida (Bastos-Formighieri \& Pasian, 2012).

Aspectos positivos como a maturidade, a sabedoria e a experiência de vida dos adultos mais velhos precisam ser valorizados (Baltes $\&$ Baltes, 1990; Garcia-Santos et al., 2012; Neri \& Vieira, 2013). Nesse sentido, é necessário otimizar recursos pessoais e sociais para enfrentar a senescência que pode resultar em alterações nas motivações dos idosos para contatos sociais (Carneiro \& Falcone, 2013; Carneiro et al., 2007; Del Prette \& Del Prette, 2005).

As HS por relacionarem-se à saúde mental, à maior satisfação pessoal, profissional, à diminuição de stress e ao incremento da qualidade de vida (Angélico et al., 2012; Braz et al., 2013; Guilland \& Monteiro, 2010; Pinto \& Barham, 2014), vem sendo estudada em todos os contextos: hospitalar, escolar, familiar e do trabalho (Braz et al., 2013; Del Prette \& Del Prette, 2013; Di Domenico-Grazziotin \& Scortegagna, 2012, 2013; Feitosa et al., 2012). Verifica-se também, estudos que demonstram a importância deste constructo na prevenção de transtornos mentais (Del Prette et al., 2013) e mais recentemente, para um envelhecimento humano com qualidade de vida (Braz et al., 2013; Braz et al., 2011; Carneiro \& Falcone, 2013; Carneiro et al., 2007).

Em relação à comunicação e desenvoltura social (F3), os participantes adultos idosos permaneceram um pouco abaixo da média e os adultos demonstraram valores acima da média. Houve uma correlação significativa negativa e baixa entre HS e maior idade $(r=0,259)$. Constata-se que quanto mais a idade avança, mais a habilidade de conversação e desenvoltura social (F3) tende a declinar. Tal tendência pode ser decorrente do fato de muitos idosos passarem a se concentrar na autoconservação dos aspectos físicos e mentais, e a reduzir os investimentos sociais e interpessoais (McAdams \& Olson, 2010; O’Brien et al., 2013).

Ainda que os escores indiquem um reper-tório menos desenvolvido da habilidade de conversação e desenvoltura social, que, retrata, por exemplo, a capacidade de lidar com situações sociais de aproximação, pedir favor, recusar pedidos abusivos (Del Prette \& Del Prette, $2005,2013)$ é importante analisar o desvio padrão do grupo dos adultos idosos $(D P=36,10)$, e dos adultos $(D P=33,99)$ que demonstra heterogeneidade. Observa-se, que apesar dos integrantes do grupo de adultos idosos terem demonstrado resultados abaixo da média não houve um padrão de comportamento, com evidências de potencial nessa habilidade entre alguns desses participantes. 
Em contrapartida, embora os participantes adultos apresentaram resultados acima da média e demonstraram um potencial elaborado, também não apresentaram homogeneidade, pois alguns indivíduos denotaram limitações. $\mathrm{Na}$ compreensão de tais achados, é necessário considerar que além da idade, outros componentes como gênero, escolaridade, situação socioeconômica, cultural e ocupacional (Del Prette \& Del Prette, 2011; Neri \& Vieira, 2013) e os traços de personalidade podem influenciar o comportamento (Caballo, 2008; Garcia-Santos et al., 2012).

No F5, que demonstra o controle da raiva e da agressividade frente ao outro em situações aversivas, observa-se uma correlação significativa negativa e moderada com a maior idade $(r=0,490)$, o que revela que à medida que a idade aumenta, essa habilidade tende a apresentar-se menos desenvolvida e a diminuir. Esse resultado acena para a possibilidade de haver um déficit nas pessoas idosas no que se refere à habilidade de autocontrole da agressividade frente a situações aversivas. A idade avançada pode trazer de uma forma mais proeminente sentimentos de medo, desespero e insegurança, o que pode contribuir para um rebaixamento do autocontrole. À medida que a idade avança, o indivíduo passa a perceber o futuro de forma restrita, pois existe um limite de tempo para a obtenção do que deseja e, para a própria vida (Neri \& Vieira, 2013).

Ainda que os idosos tenham demonstrado menores níveis nas HS em comparação aos não idosos, é possível promulgar as potencialidades e superar os aspectos distônicos desta fase (Braz et al., 2011; Carneiro \& Falcone, 2013; Del Prette \& Del Prette, 2011). Eles têm capacidade de integrar o passado, ser produtivo no presente, auxiliar as novas gerações (Baltes \& Baltes, 1990; Braz et al., 2013; Garcia-Santos et al., 2012), mas é preciso que a sociedade ofereça condições para isso e reconheça a sua importância (Braz et al., 2011; Carneiro et al., 2007; Neri \& Vieira, 2013).

Indo um pouco mais além é necessário avanços no campo da psicologia, especialmente no estudo dos adultos mais velhos, dos longe- vos, e no desenvolvimento de medidas de avaliação que possam responder as demandas desta população. Há necessidade de construção de instrumentos de avaliação psicológica, pois esta população mostra-se diferenciada em contextos diversos, e em termos de HS (Braz et al., 2013; Braz et al., 2011; Carneiro \& Falcone, 2013; Del Prette \& Del Prette, 2013).

Como se pode constatar, os resultados desta pesquisa trouxeram achados importantes, na medida em que elucidaram características das HS dos adultos idosos, quando comparados com adultos. Entre as limitações a serem consideradas no alcance dos resultados, destaca-se que a pesquisa utilizou um delineamento transversal e correlacional e, por isso, as análises estatísticas realizadas não permitem conclusões causais, apenas indicam possíveis associações entre as variáveis.

Em relação às contribuições, destaca-se que a pesquisa procurou investigar dimensões positivas do envelhecimento, como as HS, o que é pouco usual na literatura, principalmente com as pessoas idosas. Foi possível verificar que na idade mais avançada ocorreu déficits nas HS, o que impulsiona a implementação de ações em benefício da saúde desta população, e a relevância da construção de um instrumento de HS com normas específicas. Por fim, considerando que outras questões como escolaridade, nível socioeconômico, gênero, estilo de vida e a personalidade, podem exercer influência nas competências sociais, e precisam ser constantemente examinadas, há necessidade de futuros estudos com amostras mais representativas, de outras regiões, e de centros de grande porte.

\section{Referências}

American Psychological Association. (2014). Guidelines for psychological practice with older adults. American Psychologist, 69(1), 34-65. doi: $10.1037 / \mathrm{a} 0035063$

Angélico, A. P., Crippa, J. A. S., \& Loureiro, S. R. (2012). Utilização do Inventário de Habilidades Sociais no diagnóstico do transtorno de ansiedade social. Psicologia: Reflexão e Crítica, 25(3), 467476. doi:10.1590/S0102-7972201200030 0006 
Baltes, P., \& Baltes, M. (1990). Psychological perspectives on successful aging: The model of selective optimization with compensation. In P. Baltes \& M. Baltes (Eds.), Successfulaging (pp. 1-34). New York: Cambridge University Press.

Bandeira, M., Costa, M. N., Del Prette, Z. A. P., Del Prette, A., \& Gerk-Carneiro, E. (2000). Qualidades psicométricas do Inventário de Habilidades Sociais (IHS): Estudo sobre a estabilidade temporal e a validade concomitante. Estudos de Psicologia (Natal), 5(2), 401-419. doi:10.1590/ S1413-294X2000000200006

Bastos-Formighieri, M. de S., \& Pasian, S. R. (2012). O Teste de Pfister em idosos. Avaliação Psicológica, 11(3), 435-448.

Braz, A. C., Cômodo, C. N., Del Prette, Z. A. P., Del Prette, A., \& Fontaine, A. M. G. (2013). Habilidades sociales e intergeneracionalidad en las relaciones familiares. Apuntes de Psicología, 31(1), 77-84.

Braz, A. C., Del Prette, Z. A. P., \& Del Prette, A. (2011). Assertive social skills training for the elderly. Behavioral Psychology/Psicología Conductual, 19(2), 373-387.

Caballo, V. E. (2008). Conceitos atuais sobre os transtornos da personalidade. In V. E. Caballo (Ed.), Manual de transtornos de personalidade: Descrição, avaliação e tratamento (pp. 25-56). São Paulo, SP: Santos.

Carneiro, R. S., \& Falcone, E. M. O. (2013). O desenvolvimento das habilidades sociais em idosos e sua relação na satisfação com a vida. Estudos de Psicologia (Natal), 18(3), 517-523. doi:10.1590/ S1413-294X2013000300012

Carneiro, R. S., Falcone, E. M., Clark, C., Del Prette, Z., \& Del Prette, A. (2007). Qualidade de vida, apoio social e depressão em idosos: Relação com habilidades sociais. Psicologia: Reflexão e Crítica, 20(2), 229-237. doi:10.1590/S010279722007000200008

Del Prette, Z. A. P., \& Del Prette, A. (2001). Inventário de Habilidades Sociais (IHS - Del- Prette): Manual de aplicação, apuração e interpretação. São Paulo, SP: Casa do Psicólogo.

Del Prette, Z. A. P., \& Del Prette, A. (2005). Inventário de Habilidades Sociais (IHS Del Prette): Manual de aplicação, apuração e interpretação (3. ed.). São Paulo, SP: Casa do Psicólogo.

Del Prette, Z. A. P., \& Del Prette, A. (2011). Psicologia das habilidades sociais: Terapia, educação e trabalho (8. ed.). Petrópolis, RJ: Vozes.
Del Prette, Z. A. P., \& Del Prette, A. (2013). Social Skills Inventory (SSI-Del-Prette): Characteristcs and studies in Brazil. In F. Osório (Ed.), Social Anxiety Disorders: From theory to practice (pp. 49-62). New York: New Science.

Del Prette, Z. A. P., Falcone, E. M. O., \& Murta, S. G. (2013). Contribuições do campo das habilidades sociais para compreensão, prevenção e tratamento dos transtornos da personalidade. In L. F. Carvalho \& R. Primi (Eds.), Perspectivas em psicologia dos transtornos da personalidade: Implicações teóricas e práticas (pp. 326-358). São Paulo, SP: Casa do Psicólogo.

Di Domenico-Grazziotin, J. B., \& Scortegagna, S. A. (2012). Zulliger Habilidade Social: Evidências de validade no contexto empresarial. Psicologia: Reflexão e Crítica, 25(1), 69-78. doi:10.1590/ S0102-79722012000100009

Di Domenico-Grazziotin, J. B., \& Scortegagna, S. A. (2013). Relacionamento interpessoal, produtividade e habilidades sociais: Um estudo correlacional. Psico-USF, 18(3), 491-500. doi:10.1590/ S1413-82712013000300015

Feitosa, F. B., Del Prette, Z. A. P., \& Del Prette, A. (2012). Social skills and academic achievement: The mediating function of cognitive competence. Temas em Psicologia, 20(1), 61-70.

Garcia-Santos, S. C., Almeida, L. da S., \& Werlang, B. S. G. (2012). Excelência humana: A contribuição da personalidade. Paidéia (Ribeirão Preto), 22(52), 251-259. doi:10.1590/S0103$-863 \times 2012000200011$

Guilland, R., \& Monteiro, J. K. (2010). Jovem em situação de desemprego: Habilidades sociais e bem-estar psicológico. Psicologia: Teoria e Prática, 12(3), 149-163.

Instituto Brasileiro de Geografia e Estatística. (2010). Censo Demográfico de 2010. Recuperado em http://www.ibge.gov.br/home/ presidencia/noticias/noticia_visualiza.php?id_ noticia $=1866 \&$ id_pagina $=1$

McAdams, D. P., \& Olson, B. D. (2010). Personality development: Continuity and change over the life course. Annual Review of Psychology, 61, 517-542. doi:10.1146/annurev. psych.093008.100507

Neri, A. L., \& Vieira, L. A. M. (2013). Envolvimento social e suporte social percebido na velhice. Revista Brasileira de Geriatria e Gerontologia, 16(3), 419-432. doi:10.1590/S180998232013000300002 
O’Brien, E. D., Konrath, S. H., Gruhn, D., \& Hagen, A. L. (2013). Empathic concern and perspective taking: Linear and quadratic effects of age across the adult life span. The Journals of Gerontology, Series B: Psychological Sciences and Social Sciences, 68(2), 168-175. doi:10.1093/ geronb/gbs055

Pinto, F. N. F. R., \& Barham, E. J. (2014). Habilidades sociais e estratégias de enfrentamento de estresse: Relação com indicadores de bem-estar psicológico em cuidadores de idosos de alta dependência. Revista Brasileira de Geriatria e Gerontologia, 15(3), 635-655. doi:10.1590/18099823.2014.13043
Schlösser, A., Rosa, G. F. C., \& More, C. L. O. O. (2014). Revisão: Comportamento suicida ao longo do ciclo vital. Temas em Psicologia, 22(1), 133-145. doi:10.9788/TP2014.1-11

United Nations. (2012). World population prospects: The 2010 revision. Retrieved from http://esa. un.org/wpp/P-WPP/htm/PWPP_PopulationAge65 Plus. htm

Recebido: 14/10/2014

$1^{a}$ revisão: 08/04/2015 Aceite final: 22/05/2015 